\title{
Anatomic direction of entry for botulinum toxin injection to treat the adductor spasmodic dysphonia in Thais
}

\author{
Patchareeporn Saeseowa ${ }^{\mathrm{a}}$ Akkararat Sartsungnern ${ }^{\mathrm{a}}$, Supaporn Srirompotonga, Kowit Chaisiwamongkol, \\ Thanarat Chantaupalee ${ }^{\mathrm{b}}$ \\ ${ }^{a}$ Department of Otolaryngology, ${ }^{b}$ Department of Anatomy, Faculty of Medicine, Khon Kaen University, \\ Khon Kaen 40002, Thailand
}

\begin{abstract}
Background: Botulinum toxin injections into the thyroarytenoid (TA) muscle of the larynx is the most popular treatment for adductor spasmodic dysphonia. Injection is usually done by percutaneous transcricothyroid membrane with either electromyography (EMG) or fiberoptic laryngoscopy (FOL) to verify placement of the needle within the TA muscle. This procedure requires a working knowledge of three-dimensional anatomy of the larynx to establish the direction for the accurate placement of the needle.

Objective: Find out the appropriate angles and depth of the needle for placement of percutaneous transcricothyroid membrane method of botulinum toxin injection by means of studying the larynges of Thai cadavers.

Methods: The descriptive study was performed in 45 Thai freshly thawed cadavers. The angle of the needle from midline sagittal plane, the angle in superior relation to tracheal plane, and the depth from midline cricothyroid (CT) membrane to midlength of TA muscle were measured from the two views of photographs, anteroposterior and lateral.

Results: The mean angle of $24.2 \pm 6.76^{\circ}$ (mean \pm SD) from the midline sagittal plane in male and $24.9 \pm 7.6^{\circ}$ in female were worked out. A mean angle in superior relation to the tracheal plane was $47.7 \pm 7.8^{\circ}$ and $51.4 \pm 9.6^{\circ}$ in male and female, respectively. The mean depth was $1.7 \pm 0.2$ and $1.4 \pm 0.1 \mathrm{~cm}$ in male and female, respectively.

Conclusion: The mean angles and depth of the needle insertion from the midline of CT membrane to the center of TA muscle in Thai laryngeal specimens were evaluated. These values were different from the studies in Caucasians, but it could provide a direct relationship to the build of the races. This knowledge may help laryngologists do this procedure more accurately with better outcome, especially in hospitals that have no EMG or FOL guide.
\end{abstract}

Keywords: Anatomic direction of entry, botulinum toxin, spasmodic dysphonia, Thais

Spasmodic dysphonia (SD) is a voice disorder characterized by effortful, strained/strangled voice quality with frequent voice breaks. [1] There are multiple treatment modalities, including voice therapy, psychological counseling, acupuncture, and pharmacotherapy [2].

Botulinum toxin has been used to treat a wide range of dystonias including blephalospasm, hemifacial spasm, oromandibular dystonia, spastic torticollis, and laryngeal dystonia. Blitzer et al. [3] reported using

Correspondence to: Assist Prof Patchareeporn Saeseow, MD, Department of Otolaryngology, Faculty of Medicine, Khon Kaen University, Khon Kaen 40002, Thailand. E-mail: raungji@ yahoo.com botulinum toxin for SD with significant improvement of voice in 1986. Since then, botulinum toxin injections into the affected laryngeal musculature have become a treatment of choice for the patients with SD.

Most of the SD is of adductor form, and the affected laryngeal musculature is thyroarytenoid (TA) muscles. There are several techniques for injecting botulinum toxin into the TA muscle. The most popular technique is the percutaneous transcricothyroid membrane with electromyographic (EMG) guidance. This technique is done under EMG guidance. The needle is passed though the midline of cricothyroid (CT) membrane, being angled superiorly and laterally in the direction of the TA muscle. 
The most effective diffusion of the chemodenervation to the motor end plates has been shown as the diffusion distribution throughout the TA muscle [4]. For this reason, the accurate placement of the needle tip in the mid TA muscle is desirable. Therefore, the knowledge of the angle and depth of the needle is important.

There are some reports regarding Caucasians [58], but no data are available for Asian especially Thai populations. In this study, we examined the appropriate angle and depth of the needle placement for percutaneous transcricothyroid membrane method of botulinum toxin injection by means of the dissection of Thai larynges.

\section{Materials and methods}

This study was performed in 45 Thai fresh thawed cadavers, donated to Anatomy Department, Faculty of Medicine, Khon Kaen University, Thailand between June 2007 and September 2008. All of the cadavers had no macroscopic pathology on the neck region. They consisted of 27 males and 18 females, with age between 29-83 years.

On these 45 cadavers, we dissected 45 larynges from the level of upper border of the hyoid to the upper trachea.

A 20-gauge needle was placed through the midline of CT membrane of the laryngeal specimen directed to the center of TA muscle (Fig. 1).

The specimen was photographed from two views: anteroposterior and lateral, as shown in Fig. 2 and Fig. 3, respectively. These images were used to measure the angle from the midline sagittal plane, the angle in superior relation to tracheal plane, and the depth of the needle from CT membrane to TA muscle.

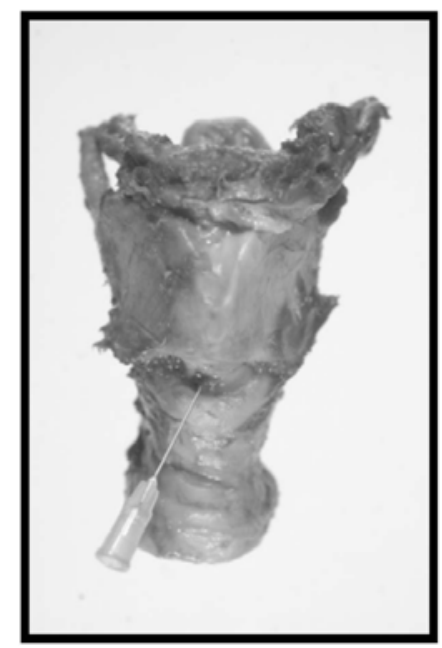

Fig. 1 A 20-gauge needle was placed through the midline of CT membrane of the laryngeal specimen directed to the center of TA muscle.

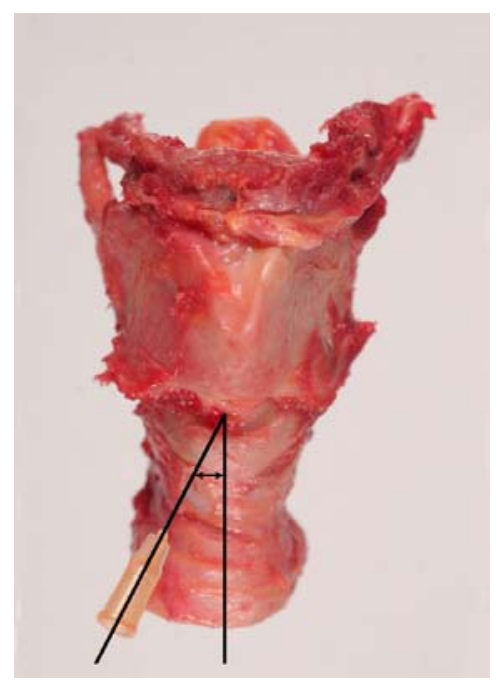

Fig. 2 The angle of needle from the midline sagittal plane was measured by the anteroposterior view of laryngeal photograph. 


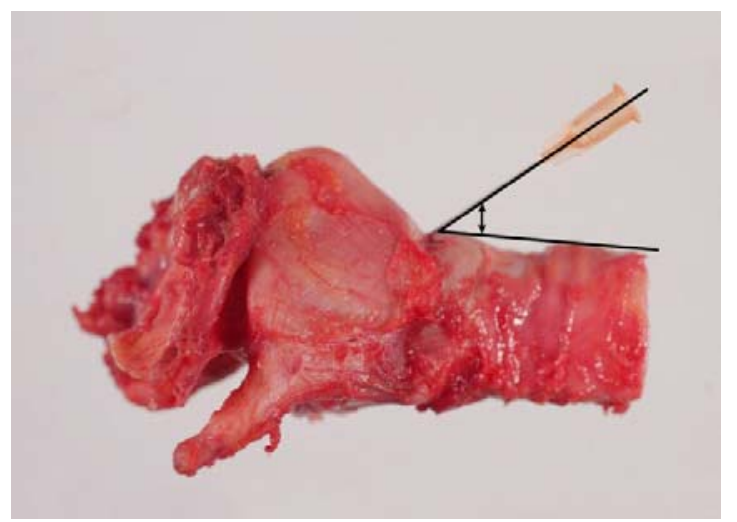

Fig. 3 The angle of needle in the superior relative to the trachea was measured by the lateral view of laryngeal photograph.

\section{Results}

The anteroposterior photographs demonstrated a mean angle of $24.2 \pm 6.7^{\circ}$ (mean $\pm \mathrm{SD}$ ) from the midline sagittal plane in male and $24.9 \pm 7.6^{\circ}$ in female. The lateral photographs demonstrated a mean angle of $47.7 \pm 7.8^{\circ}$ in male in the superior relative to the tracheal plane and $51.4 \pm 9.6^{\circ}$ in female. The mean depth was $1.7 \pm 0.2$ and $1.4 \pm 0.1 \mathrm{~cm}$ in male and female, respectively.

\section{Discussion}

Although there is no cure for SD, intralaryngeal injection of botulinum toxin has proven to be a safe and effective treatment. There are several techniques for injections such as oral injection with an indirect laryngoscopic approach [9], transcutanous under fiberoptic laryngoscopy (FOL) guide [9, 10], and transcutanous under EMG guide [11-13].

We prefer the percutaneous transcricothyroid membrane with EMG guidance because it takes less time, causes less discomfort, and is acceptable to virtually all patients. However, physicians should know the accurate placement of the needle because it will take less time to do the procedure, and decrease tissue trauma and bleeding. Repeated insertion of the needle may increase the chance of leakage of the botulinum toxin into the airway. Therefore, one point insertion should be ideal for the injection.

The placement of the needle was described in some previous researches. Yin et al. [6] inserted the needle electrode $0.2-0.3 \mathrm{~cm}$ lateral to the midline of CT membrane to avoid entering the airway and then directed the needle posteriorly, superiorly, and laterally at an angle of $45^{\circ}$ with a depth of 2.5-3.5 cm. Gibbs and Blitzer [7] advanced the needle from midline of CT membrane to TA muscle, then angled up approximately $30^{\circ}$ and approtimately $30^{\circ}$ laterally. Munin et al. [8] used the angle of $45^{\circ}$ superiorly and $20^{\circ}$ laterally with the approximate depth of $2 \mathrm{~cm}$. Castellanos et al. [5] reported a mean angle of $12 \pm 2.8^{\circ}$ (mean \pm SD) laterally and $69 \pm 5.2^{\circ}$ superiorly in relation to the thyroid lamina/notch and an angle of $50 \pm 4.3^{\circ}$ superiorly from the trachea.

Our results were different from the previous studies performed on the Caucasians. This may be because of the build of different races in which the size and shape could be differently smaller in Thais. The values obtained are more relevant in the Thai patients than the Caucasian data. The data reported here could help the laryngologist do this procedure more accurately with one point insertion and have a better outcome especially in the hospital that there was no EMG or FOL guide.

The disadvantage of the botulinum toxin injection is its short period of function; approximately 3-6 months. The surgical treatments intended to provide long-term symptom control have been advancing over recent years. Nakamura et al. [14] reported successful treatment with bilateral TA myectomy (Muta method) in 2008 .

Most medical and surgical treatments have been aimed at denervation of the laryngeal muscles to block symptom expression in the voice, and have both adverse effects as well as benefits. Further research is needed regarding the target therapy towards the central neurological abnormality [15].

In conclusion, we evaluated the mean angles and depth of the needle insertion from the midline of CT membrane to the center of TA muscle in Thai laryngeal specimens. These values were different from the studies in Caucasians, but it could provide a direct relationship to the build of the races, which may help 
the laryngologist do this procedure in Thai patients more accurately with better outcome, especially in the hospitals that have no EMG or FOL guide.

\section{Acknowledgement}

This study was financially supported by the Faculty of Medicine, Khon Kaen University. The authors thank Dr. Somboon Srungboonmee (Emeritus Professor of Anatomy, Mahidol University and Foundation Chairman of KKU Department of Anatomy) for his revision of the manuscript and $\mathrm{Mr}$. Charles Taylor MT (ASCP) for his proof-reading this manuscript.

\section{References}

1. Rubin JS, Sataloff RT, Kovovin GS. Diagnosis and treatment of voice disorder. $2^{\text {nd }}$ ed. New York:ThomsonDelmar Learning; 2003. p. 416.

2. Grillone GA, Chan T. Laryngeal dystonia. Otolaryngol Clin NAm. 2006; 39:87-100.

3. Bliter A, Brin MF, Faln S, Lange D, Lovelace RE. Botulinum toxin (Botox) for the treatment of "spastic dysphonia" as part of a trial of toxin injection for the treatment of other cranial dystonias. Laryngoscope. 1986; 96:1300-1.

4. Rosen M, Malmgren LT, Gacek RR. Three-dimensional computer reconstruction of the distribution of neuromuscular junctions in the thyroarytenoid muscle. Ann Otol Rhinol Laryngol. 1983; 92:424-9.

5. Castellanos PF, Gates GA, Esselman G, Song F, Vannier MW, Kuo M. Anatomic considerations in botulinum toxin type A therapy for spasmodic dysphonia. Laryngoscope. 1994; 104:656-62.

6. Yin SS, Qiu WW, Strucker FJ. Major patterns of laryngeal electromyography and their clinical application. Laryngoscope. 1997; 107:126-36.

7. Gibbs SR, Blitzer A. Botulinum toxin for the treatment of spasmodic dysphonia. Otolaryngol Clin NAm. 2000; 33:879-94.

8. Munin MC, Murry T, Rosen CA. Laryngeal electromyography diagnosis and prognostic applications. Otolaryngol Clin N Am. 2000; 33:759-70.

9. Ford CN, Bless A, Lowery JD. Indirect laryngoscopic approach for injection of botulinum toxin in spasmodic dysphonia. Otolaryngol Head Neck Surg. 1990; 103: 752-8.

10. Klap P, Marion MH, Perrin A, Fresnel-Elbaz E. Treatment of spasmodic dysphonia with botulinum toxin. Ann Otolaryngol Chir Cervicofac. 1991; 108: 477-82.

11. Green DC, Berke GS, Ward PH, Gerratt BR. Point-touch technique of botulinum injection for the treatment of spasmodic dysphonia. Ann Otol Rhinol Laryngol. 1992; 101: 883-7.

12. Blitzer A, Brin MF, Stewart CF. Botulinum toxin management of spasmodic dysphonia (laryngeal dystonia): a 12 year experience in more than 900 patients. Laryngoscope. 1998; 108:1435-41.

13. Srirompotong S, Saeseow P, Taweesaengsuksakul R, Kharmwan S. Botulinum toxin injection for treatment of spasmodic dysphonia: experience at Srinagarind hospital. J Med Assoc Thai. 2006; 89:2077-80.

14. Nakamura K, Muta H, Watanabe $\mathrm{Y}$, Mochizuki R, Yoshida T, Suzuki M. Surgical treatment for adductor spasmodic dysphonia-efficacy of bilateral thyroarytenoid myectomy under microlaryngoscopy. Acta Oto-Laryngologica. 2008; 128:1348-53.

15. Ludlow CL. Treatment for spasmodic dysphonia: limitations of current approaches. Curr Opin Otolaryngol Head Neck Surg. 2009; 17:160-5. 